\title{
Capítulo 5 YouTube en Ecuador: diagnóstico y expectativas
}

\author{
Diana Rivera-Rogel \\ Universidad Técnica Particular de Loja (Ecuador) \\ Orcid: https://orcid.org/oooo-ooo1-8965-0170 \\ Leonardo Ordoñez Álvarez \\ Universidad Politécnica Salesiana (Ecuador) \\ Orcid: https://orcid.org/oooo-ooo2-3967-02OX \\ DOI: https://doi.org/ 10.16921/ciespal.14.7
}

\section{Resumen}

En la actualidad los contenidos sociales, musicales y de entretenimiento acaparan la atención de los usuarios que han encontrado material de apego, afinidad e incluso la posibilidad de replicar lo que se ve y compartirlo con otros usuarios. En este sentido, YouTube se ha convertido en la segunda plataforma digital más utilizada a nivel mundial y también en Ecuador, ha desplazado en cierta medida a las industrias culturales audiovisuales tradicionales y ha dado paso a los denominados YouTubers que cada vez ganan más suscriptores y de a poco fortalecen su modelo de negocio. Aunque las conclusiones de esta investigación son de carácter general, reafirman que los usuarios prefieren cada vez más el contenido digital, están exigiendo mayor diversidad, sin horarios ni programación.

Palabras clave: YouTube; Ecuador; redes sociales, plataformas; video; audiovisual. 


\begin{abstract}
Nowadays, social, musical and entertainment contents attract the attention of users who have found material of attachment, affinity and even the possibility of replicating what is seen and sharing it with other users. In this sense, YouTube has become the second most used digital platform worldwide and also in Ecuador, has displaced to some extent the traditional audiovisual cultural industries and has given way to the so-called YouTubers that are gaining more and more subscribers and gradually strengthening their business model. Although the conclusions of this research are general, they reaffirm that users increasingly prefer digital content, and are demanding greater diversity, without schedules or programming.
\end{abstract}

Keywords: YouTube; Ecuador; social networks, platforms; video; audiovisual

$\mathrm{Al}$ igual que otros medios/plataformas digitales YouTube se presenta como un canal de video en constante actualización. Los usuarios pueden participar de forma activa a través de la creación de material, puntuando, comentando o integrando contenido en otras plataformas como las redes sociales, blogs, etc. No se trata de la única plataforma online de contenido audiovisual, pero sí es la más utilizada. Según el ranking Alexa Ecuador, YouTube se ubica en el segundo lugar después de Google ${ }^{2}$.

YouTube es un sitio web o plataforma digital que permite subir, compartir y mirar videos. Nace en febrero de 2005, de la mano de Steve Chen, Chad Hurley y Jawed Karim; tres ex empleados de la compañía PayPal quienes dan vida a YouTube que, en 2006, cuando ya contaba con millones de usuarios sería adquirida por Google al desembolsar 1.650 millones de dólares (Murolo y Lacorte, 2015).

YouTube se creó con el slogan Broadcast Yourself, que en español significa "trasmite tú mismo", que a decir de Antolín (2012) esta frase indica el paso que se daría con YouTube, en cuanto a la revolución del

1. Fecha de consulta: 28 de febrero de 2020. 
video y de la televisión, donde es el usuario quien decide que ver eincluso tiene la opción de sugerir contenido a otros. En este sentido, YouTube representa así los paradigmas de la digitalización del audiovisual, llegando a ser en parte, junto a otras aplicaciones, responsable de la actual crisis de las industrias culturales en el ámbito audiovisual, especialmente de la televisión tradicional. YouTube es calificada como una de las redes sociales más populares y con más aceptación alrededor del mundo, considerando que brinda la oportunidad al usuario de acceder a libertad de diversos tipos de contenidos audiovisuales, entre ellos, videos de: música, charlas, talleres, películas, documentales, archivos periodísticos, dibujos animados, reportajes, fragmentos de series, etc.

Bañuelos (2009) se refiere a YouTube como la plataforma de la sociedad del espectáculo, donde "se imponen nuevas formas de recepción y producción de sentido en la noción, forma y contenido del espectáculo y otras características de la industria cultural". Lo señalado se percibe en la presencia de músicos, artistas, actores y otros personajes, que utilizan esta plataforma para exponer sus productos artísticos, y llevarlos a viralizar con el apoyo de otras redes sociales. Según Rivera-Rogel, Velásquez y B-Flandoli (2018) "YouTube es una plataforma digital donde los usuarios pueden subir y compartir videos de manera fácil, cómoda y rápida, interactuando con miles de personas en diferentes partes del mundo".

El avance y actualización de la tecnología, así como el cambio en los medios de comunicación posibilitan que YouTube sea una herramienta de alta usabilidad. "Ya no es necesario llegar a casa para encender la televisión y mirar los acontecimientos del día, cuando en la calle algún circunstancial testigo ya los grabó y seguramente subió a YouTube" (De Cicco, 2009, p.31). Complementando el párrafo anterior, Pérez Rufí (2011, p. 161) mencionan que YouTube ha dejado de responder el concepto de "tu televisión", convirtiéndose en un canal comercial más, una televisión a la carta que, efectivamente, permite un feedback con los vídeos y la divulgación de contenidos propios. 
La actualización de contenidos en esta plataforma se desarrolla de forma continua y el incremento de usuarios que comparten sus productos también evidencia crecimiento, es por ello que Mejía (2019) señala que YouTube alcanzó, en enero de 2019, mil novecientos millones de usuarios activos en un mes, traduciéndose también en un significativo número de videos para observar. De acuerdo con Alexa (2020) es el segundo sitio más visitado del mundo, después de Google. El crecimiento de YouTube se debe principalmente a la interacción que ha logrado entre usuarios, en la actualidad dicha interacción es más efectiva, sobre todo porque permite realizar videos en directo y los usuarios pueden comentar mientras el video se está reproduciendo, generar un "me gusta", "no me gusta" o en su defecto compartir.

Esta participación de los usuarios puede ser muy variable, MontesVozmediano, García-Jiménez y Menor-Sendra (2018) aseguran que, por ejemplo, en ámbitos políticos no se visualiza acciones masivas de producción por parte de los usuarios, es preferible para ellos compartir videos ya publicados por otros medios de comunicación, que crearlos; sin embargo, los usuarios más activos están presentes creando contenido relacionado a distintos ámbitos.

Con relación a las plataformas de descargas de videos, según Mascheroni y Olafsson (2014) "YouTube es una de las más utilizadas, alrededor del $55 \%$ de los jóvenes europeos la usan preferentemente.

En este capítulo, intentamos hacer un acercamiento a la evolución de la plataforma YouTube en Ecuador y sus principales vertientes de crecimiento y uso por parte de los usuarios ecuatorianos.

\section{De la televisión tradicional a YouTube}

Desde la primera publicación realizada el 23 de abril de 2005, la plataforma de YouTube ha sido una constante evolución, al año de haber sido creada, en el 2006 fue adquirida por Google, quien analizó su potencial, su fortaleza y visión para el futuro. Luego de quince años, encabeza la lista de páginas de visualización de videos en el mundo. 
Según sus estadísticas, YouTube tiene más de dos millones de usuarios, entre 18 a 34 años como target central, disponible en cien países con ochenta idiomas, en cuanto a las horas de reproducción, calculan mil millones de horas diarias. Pero un dato en particular sobresale, cuando menciona el target, "YouTube, solo en su versión móvil, llega a más personas que cualquier canal de TV en EE.UU.” (YouTube, 2020).

Esta mención es clara, su competencia directa es la televisión tradicional, y esa es la nueva apuesta de esta plataforma, en el 2019, realizó el lanzamiento de YouTubeTV y la preferencia del público norteamericano donde al momento funciona la aplicación, ha sido favorable, pero en qué se basa el éxito que tiene YouTube.

Los internautas lo han conceptualizado de la siguiente manera; "se puede ver lo que se quiere, cuando se quiere", con esta expresión, rompe la costumbre de una regla en la televisión tradicional, ver un programa a una determinada hora y día, eliminando así la programación y la clasificación de audiencias como lo determina incluso la Ley de Comunicación en el Ecuador en el Art. 65:

Se establece tres tipos de audiencias con sus correspondientes franjas horarias, tanto para la programación de los medios de comunicación de radio y televisión, incluidos los canales locales de los sistemas de audio y video por suscripción, como para la publicidad comercial y los mensajes del Estado (Ley Orgánica de Comunicación, 2013)

Siendo la Familiar de 06:00 a 18:00 con clasificación A, Apto para todo Público, Responsabilidad compartida de 18:00 a 22:00 con clasificación B y la C que es público adulto de 22:00 a 06:00 del siguiente día. (pág. 12).

Esta estratificación que servía para la programación se ven vulneradas con la propuesta de YouTube, quien desde sus inicios pretendió diversificar las audiencias según los gustos de los usuarios, en la actualidad con la inteligencia artificial, la plataforma reordena los contenidos para cada usuario o cada vez que se abre una nueva sesión, los parámetros se ajustan. 
Salar Kamangar CEO de YouTube (2010-2014), mencionó su idea de canalizar contenidos en base a lo que hace la televisión tradicional, que es tener programas por géneros, pero el objetivo es que los usuarios no solo vean un video enviado por un contacto, sino que se quede a visualizar el resto de los contenidos que posee ese canal.

Las agencias de publicidad ven con agrado esta nueva manera de disgregación, ya que, al ajustar los contenidos por usuario, sus campañas se ven mejor direccionadas y personalizadas, los productos son presentados a quienes tienen interés sobre ellos, mientras que la televisión, solo mira como una masa en el universo de espectadores.

A todo esto, se suma que los televidentes dependen del aparato televisor para ver sus programas, en la actualidad y con los avances tecnológicos, hay más dispositivos celulares inteligentes que permiten visualizar todo tipo de contenidos y por fábrica viene incluido YouTube y en los últimos años con los Smart tv, la aplicación también viene incluida, lo que ha provocado que canales de la televisión tradicional migren a esta plataforma y rediseñen sus contenidos como lo ha realizado Telecinco de España con Mediaset, que propuso a sus usuarios tradicionales y digitales su pull de canales con contenidos específicos y series con episodios continuos, algo que el espectador actual aprecia y no espera una semana para ver el siguiente capítulo, datos de diciembre 2019, publicados por ComScore (2020), Mediaset España duplica su consumo registrado en el 2018, 4778 millones de videos vistos colocándolo como un medio líder en consumo digital con programas de la televisión tradicional.

Otro factor que aporta al éxito de YouTube, es que los televidentes visualizan más contenidos por Internet, que la televisión y está aún vive porque existe fieles televidentes que sobrepasan los 60 años (2017), pero el cambio generacional se viene y el rey será la pantalla chica, un dispositivo móvil que también se prepara para destronar a la televisión tradicional.

El estudio realizado por Ericsson ConsumerLab TV and Media de 2017, ya denota el cambio en el hábito de ver televisión, 6 de cada 10 
consumidores prefieren ver televisión por compra o demanda que por sistema tradicional y para el 2020 se espera que este aumente con un alcance del 65\% mirando programación más de 25 horas por semana (Ericsson Consumer, 2017). Este mismo estudio señala que este 2020, solo 1 de cada 10 consumidores se quedará viendo la televisión en una pantalla tradicional, una significativa diminución del $50 \%$ en comparación del 2010, tecnológicamente la televisión tradicional al parecer está llegando a su fin. Al parecer aquí cabe una frase con mucha historia, y usada en la época de la monarquía, en la cual se despedía al soberano fallecido e inmediatamente se reconocía a su sucesor.

"El rey ha muerto ¡Viva el Rey”!

\section{YouTube en Ecuador. Diagnóstico y expectativas}

En Latinoamérica, YouTube lidera la lista de redes sociales con el 95\% de uso, se utiliza principalmente para entretenimiento, información y educación. Aproximadamente 17 millones de habitantes de Ecuador, más de 13,8 millones están conectados y mantienen acceso a Internet. Las principales plataformas o redes sociales son Facebook y YouTube, esta última con un tiempo estimado en el sitio de 9:48 minutos al día3

YouTube, al igual que en otros países ha tomado impulso en Ecuador y con ello, la posibilidad de presentar contenido en tiempo real y a mayor escala. Esta plataforma también es utilizada por los medios de comunicación tradicionales para dar a conocer su contenido y expandirlo a una mayor audiencia y así lograr mayor impacto con sus informaciones.

YouTube, hoy en día se presenta como una "red social generadora de capital económico" (Hidalgo y Segarra, 2017, p.45), es decir, una plataforma donde determinados usuarios han creado un negocio digital

3 SemRush Top 500 páginas Ecuador consultado el 10 de enero de 2019. Por número de visitas promedio desde Google a versiones web. Infografía elaborada por Eluniverso.com en base a información de este estudio. 
compartiendo y viralizando. Esta posibilidad de generar contenido de diversa índole y darse a conocer en otras latitudes ha sido aprovechada por los YouTubers ecuatorianos, quienes han dado un giro total a las posibilidades de comunicarnos.

En Ecuador, el crecimiento de los YouTubers ha sido notorio desde el 2013. Muchos de ellos se han convertido en el nuevo emblema nacional e internacional. Hasta el 2016, para los YouTubers ecuatorianos era imposible generar ingresos a través del programa socios de YouTube, debido a que el país no formaba parte de este, fue hasta el 2017 que Ecuador fue incluido y con ello inician a monetizar su trabajo los YouTubers, este tema se puede corroborar en la página Social Blade.

La influencia de la producción de videos para la plataforma de YouTube ha ido creciendo internacionalmente y también en Ecuador. Desde acciones cotidianas, retos, hasta canales personalizados, en cocina, belleza, deportes, etc., se crean un sinnúmero de opciones para compartir con el resto de los usuarios, por lo que los jóvenes ecuatorianos han tomado este rumbo y aportan con nuevos videos, tomando los roles de directores, productores y editores creando e innovando en el contenido.

Por su parte, Diario Metro de Ecuador en una publicación de diciembre de 2019, señala a cinco YouTubers que han alcanzado un número importante de seguidores y con ello reconocimiento internacional: RaptorGamer (4,59 millones), Criss Huera (3,57 millones), Wallas Da Silva (1,46 millones), Nancy Risol (2,23 millones), Felipe Crespo (798 mil) y Enchufe Tv (22,2 millones), en comparación con la página de la productora de enchufe tv, touché films que apenas alcanza los 119.000 suscriptores.

\section{Análisis del fenómeno YouTube en Ecuador. Experiencias}

A más de los cinco YouTubers mencionados anteriormente, diario El Universo (2019), incluye a: 
Doc Tops, que se dedica a recopilar temas variados, en forma de top ten, alcanza 9,8 millones de seguidores.

Los diferentes gestores de contenido se basan en el género de entretenimiento y la comedia, pero contenidos informativos, de análisis e investigación, aparte de ser pocos los canales en esta plataforma tienen baja cantidad de seguidores. Tenemos el caso muy particular del periodista Robinson Robles, destacado productor y corresponsal de varias cadenas internacionales como Univisión, Telemundo, CNN y actualmente de HispanTV, además de ser miembro de la Academia Nacional de Historia del Ecuador, maneja un canal con su nombre desde el 14, de diciembre del 2009, llega a una cifra de apenas 27.900 suscriptores.

En el canal de Robles existen videos con un segmento denominado "Historia Viva, la memoria del Ecuador", se resume en un trabajo de 25 años de investigación y recopilación de contenido histórico audiovisual del país. Pero debido a los ataques de hackers, varios videos han sido bajados o censurados por la plataforma, en consecuencia, desde el 22 de noviembre del 2019 sus videos se alojan en VIMEO, pero pese hacer un producto de calidad técnica y con temas relevantes del país, los videos en YouTube oscilan entre 500 a 3500 visitas, si analizamos entre periodos de tiempo entre este canal y cualquiera de los seis anteriores descritos, los videos de los YouTubers en poco tiempo lo sobrepasan de manera abrumadora.

Revisando las páginas de los medios tradicionales como Teleamazonas, Ecuavisa, y Canal Uno, sumando a los tres pueden llegar a 1,3 millones de seguidores, de la misma manera los videos que estos canales suben van entre 600 a 11.000 visitas que comparados solo con un video de Nancy Risol denominado "Como enamorar a tu pareja" subido el 12 de febrero del 2020 llega a 443,398 visitas (2020), los deja muy por detrás a los medios tradicionales.

Como se puede apreciar, la diversidad del público o el universo amplio que nos permite la red, o hace que los videos informativos o de investigación se pierdan y solo sobresalgan aquellos de contenido 
superfluo, o realmente los espectadores o internautas solo buscan entreteniendo, sea cual sea su forma sin importar el fondo mucho menos un concepto audiovisual.

Tal vez, YouTube no se presta para el género de información, como si lo fue Facebook, con las transmisiones en vivo en las manifestaciones de octubre 2019 en Ecuador y donde la mayoría de ciudadanos buscaron detalles de lo que sucedía en cada rincón del país, predominando los canales digitales, ejemplo Huambra Radio, ACN entre otros, y debido a esto los medios tradicionales como Ecuavisa y Teleamazonas usaron sus fan page para llevar el minuto a minuto de lo que trascurría en la capital de la República. Por ello, se puede inferir que YouTube se orienta al entretenimiento sin importar la calidad del contenido, un mensaje, un concepto un fondo, solo un fin, entretener, como alguna vez se le dijo a la televisión "la caja tonta", al parecer hay un nuevo medio que quiere ocupar esa designación.

La página Nextv Latin America (Bertran, 2020), menciona que YouTube va a realizar la primera producción original en Ecuador de la mano con Touché Films (enchufe tv) y se encuentra ya en la etapa de realización, en las provincias de Imbabura y Pichincha con locaciones exteriores y otras en estudio, el género será comedia de acción y aventura, así lo manifestó Arturo Yépez productor ejecutivo al diario El Comercio.

Complementariamente, el estudio de Ericsson Consumer (2017) manifiesta que los cambios de actitudes del consumidor y la generación de jóvenes que está ahora en el mundo, solo busca entreteniendo sea para su momento de ocio, el trabajo o por demanda, a esto se suma que el 70\% de los consumidores miran los videos desde la pantalla del móvil, lo que hace que visualice los productos en cualquier lugar e instante. Por lo tanto, los denominados infoproductores deberán acoplarse a los nuevos procesos y nuevas exigencias de una audiencia tan diversa y fragmentada, convirtiéndose en el nuevo reto de la actualidad y del futuro. 


\section{Conclusiones}

En menos de una década, las nuevas tecnologías y plataformas se han convertido en los nuevos medios de comunicación y de entretenimiento, en ese tenor, los profesionales de la comunicación deben adaptarse a los diferentes tipos de lenguaje y procesos de producción audiovisual para satisfacer las necesidades de los consumidores de la pantalla móvil sumando la realidad virtual a los procesos, la cual se extiende a nivel mundial.

Si la televisión quiere sobrevivir deberá ofrecer contenidos para ese mega grupo diverso, y no solo como lo hace en la actualidad, coger y conectar su señal de aire a las redes sociales, no se da cuenta aún que los públicos son diferentes y que el público que le dio poder y su largo reinado está muriendo. El público actual es joven y demanda más, sin horarios ni programaciones.

En definitiva, YouTube se traduce en el consumo estereotipado, en el que los adolescentes están presentes de manera masiva -sin importar las fronteras- decidiendo cuáles son los fenómenos mediáticos, influidos por poderosas campañas que se transmiten a través de los diferentes canales digitales. En suma, Ecuador está inmerso en el mundo globalizado y YouTube es fruto de esa globalización, por ende, Ecuador y YouTube están y estarán interrelacionados por el consumo.

\section{Referencias}

Alexa. (2020). Site Info. EEUU. Alexa. Competitive Analysis, Marketing Mix, and Website Traffic. https://www.alexa.com/siteinfo

Antolín, R. (2012). YouTube como paradigma del video y la televisión en la Web 2.o. (Tesis doctoral). Universidad Complutense de Madrid, Madrid, España. Bertran, A. (24 de Enero de 2020). YouTube realiza primera producción original en Ecuador con touché films. París, Francia: NextTV News. http://nextvla- 
tam.com/YouTube-realiza-primera-produccion-original-en-ecuador-con-touche-films/

Bañuelos, J. (2009). YouTube como plataforma de la sociedad del espectáculo. Razón y Palabra (66).

ComScore (3 de Febrero de 2020). MediaSet.es. Obtenido de https://cutt.ly/nr6TWWI

De Cicco, J. (2009). YouTube, el archivo audiovisual de la memoria colectiva. Recuperado de https://cutt.ly/Ir6Y1oJ

El Universo (20 de agosto de 2019). Cinco YouTubers ecuatorianos con más de 1 millón. de fans. El Universo. Recuperado de https://bit.ly/2IBC8zl

Ericsson Consumer (diciembre de 2017). Informe Consumer lab 2017. Recuperado de https://cutt.ly/wr6TvDw

Hidalgo, T. y Segarra, J. (2017). El fenómeno YouTuber y su expansión transmedia. Análisis del empoderamiento juvenil en redes sociales. Fonseca, Journal of Communication, (15), 43-56. Recuperado de http://bit.ly/2vCqBws

Ley Orgánica de Comunicación (25 de Junio de 2013). Registro Oficial tercer Suplemento. Quito, Pichincha, Ecuador: Editora Nacional.

Mascheroni, G. y Ólafsoon, K. (2014). Net children go mobile: Risks and opportunities. Milano: Educatt.

Mejía, J. (2019). Estadísticas de redes sociales 2019: Usuarios de Facebook, Twitter, Instagram. YouTube, LinkedIn, Whatsapp y otros. Recuperado de http://bit. ly/2SKIMta

Montes-Vozmediano, M., García-Jiménez, A. y Menor-Sendra, J. (2018). Los vídeos de los adolescentes en YouTube: Características y vulnerabilidades digitales. Comunicar, 26(54), 61-69. Recuperado de http://bit.ly/2wjRGFf

Murolo, N. L. y Lacorte, N. (2015). De los bloopers a los YouTubers. Diez años de YouTube en la cultura digital. Question, 1(45), 15-29.

Pérez Rufí, J. P. (2011). YouTube ya no es" tu televisión”: cultura colaborativa y red comercial en el vídeo online. Revista Internacional de Comunicación Audiovisual, Publicidad y Literatura, 1 (9), 146-162. Recuperado de http:// bit.ly/2uDYA7V

Rivera-Rogel, D., Benavides, A. V. y B-Flandoli, A. M. (2018). YouTubers de Ecuador y Colombia y su aporte a la educación. En I. Aguaded (Presidencia), 
Comunicación-Educación para el bien vivir. Ponencia presentada en el III Congreso Internacional de Competencias Mediáticas, Medellín, Colombia. 
